\title{
Infrared Spectroscope for Electron Bunch-length Measurement: Heat Sensor Parameters Analysis.
}

\author{
Gilles Dongmo - Momo \\ Office of Science, Science Undergraduate Laboratory Internship (SULI) Program
}

Towson University

Baltimore, Maryland

SLAC National Laboratory

Menlo Park, California

$08 / 20 / 2011$

Prepared in partial fulfillment of the requirements of the Office of Science, U.S. Department of Energy's SULI under the direction of Dr. Josef Frisch at the Linac Coherent Light Source (LCLS).

Participant:

Signature

Research Advisor:

Signature 


\section{Table of Contents}

$\begin{array}{lr}\text { Abstract } & 3 \\ \text { Introduction } & 4 \\ \text { Methods } & 5 \\ \text { Results } & 9 \\ \text { Discussion and Conclusion } & 17 \\ \text { Acknowledgements } & 19 \\ \text { References } & 19\end{array}$

2|P a g e 


\begin{abstract}
:
Infrared Spectroscope for Electron Bunch-length Measurement: Heat Sensor Parameters Analysis. Gilles Dongmo - Momo (Towson University, Baltimore, MD 21252) Josef Frisch (ICD Accelerator Physics \& Engineering department, SLAC National Laboratory, Menlo Park CA 94025)
\end{abstract}

The Linac Coherent Light Source (LCLS) is used for many experiments. Taking advantage of the free electron laser (FEL) process, scientists of various fields perform experiments of all kind. Some for example study protein folding; other experiments are more interested in the way electrons interact with the molecules before they are destroyed. These experiments among many others have very little information about the electrons' $x$-ray produced by the FEL, except that the FEL is using bunches less than 10 femtoseconds long. To be able to interpret the data collected from those experiments, more accurate information is needed about the electron's bunch-length. Existing bunch length measurement techniques are not suitable for the measurement of such small time scales. Hence the need to design a device that will provide more precise information about the electron bunch length. This paper investigates the use of a pyreoelectric heat sensor that has a sensitivity of about 1.34 micro amps per watt for the single cell detector. Such sensitivity, added to the fact that the detector is an array sensor, makes the detector studied the primary candidate to be integrated to an infrared spectrometer designed to better measure the LCLS' electron bunch length. 


\section{INTRODUCTION:}

The X-rays produced by the LCLS have the same profile in the time domain as the electrons producing them. More information about the electrons can be translated to more information about the x-rays. Many techniques were developed to characterize electrons. To give accurate information about the LCLS' electron bunch, those techniques were able to approximate the electron bunch length to be shorter than 10 femto-seconds. [1] But the need to get better image resolution out of the LCLS's Free Electron Laser becomes a necessity added to the fact that current experimental efforts require a more precise measurement for LCLS electron pulse duration, new bunch-length measurement techniques are being developed[2]. An infrared spectrometer was designed as a means of indirectly measuring the pulse duration. [3]. Due to the difficulty of directly measuring the electron bunch length, measurement efforts must focus on indirectly extracting the pulse duration from other properties of the beam.

For this characterization purpose, an innovative spectrometer designed to measure the optical transition radiation spectrum of the electrons as they pass through a foil can provide such an indirect measurement. Getting the wavelength distribution of this radiation and then calculating its inverse Fourier transform will provide a space-time profile of the electron bunch. The optical transition radiation is mostly in the infrared range which makes it hard to work with, especially in the optics alignment and setup. In the final setup to be used at LCLS, the radiation produced from the electrons will go through a set of optical elements [3] including gold-coated mirrors, irises, gold-coated off-axis parabolic mirrors, and a prism to stop at a detector. [4] 


\section{METHODS:}

The project aim is to use infrared spectroscopy to get information on the electrons from the optical transition radiation (OTR) that are produced when electrons approach and pass through a

thin metal foil. [5] The ultimate spectrometer design is expected to direct the OTR beam to a prism that will disperse infrared light from about 0.6 to 40 micro meters. This covers the infrared range most relevant to the OTR emitted by the LCLS pulses. The primary setup was done on an optical bench using lasers of different wavelengths to simulate the OTR. The IR sensor is a heat detector that will be connected to a computer.

Most available spectrometers are designed to detect a particular wavelength of light or a very narrow range of wavelengths. The Spectrometer that is being designed is intended to be sensitive to a wide range of infrared light for wavelengths varying from 0.6 nanometers to 40 nanometers. The infrared spectrometer includes an optical set up that ends with an infrared detector. For this setup, a piezoelectric heat sensor was utilized. The PY-LA-S-128 from PYREOS is a line sensor array made of 128 two and a half-millimeter long sensor elements, each lined up over 14.3 millimeters. Each element of the detector constitutes a pixel and is made of a thin film of pyroelectric PZT (Lead zirconate titanate) material. PZT is a material that develops a voltage difference when experiencing a temperature, mostly infrared light change; these characteristics make this innovative sensor the perfect candidate for the project. [6] Most detectors of this type are made of a single cell element. The detector used in this experiment is sensitive a wide band in the IR spectrum. [7] 


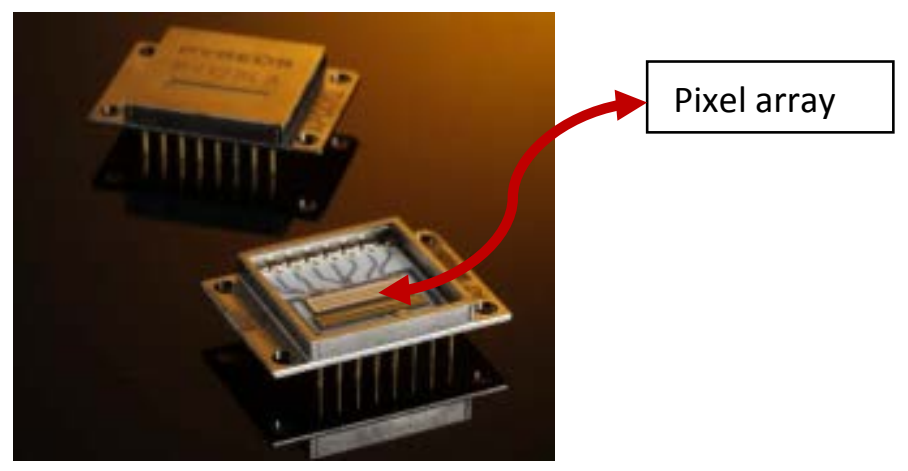

Figure 1: Line sensor.

The detector is mounted on a board on which all the electronics necessary to the data acquisition are setup. The detector was purchased with the board and the necessary software allowing us to upload collected data during the experiment. [8]

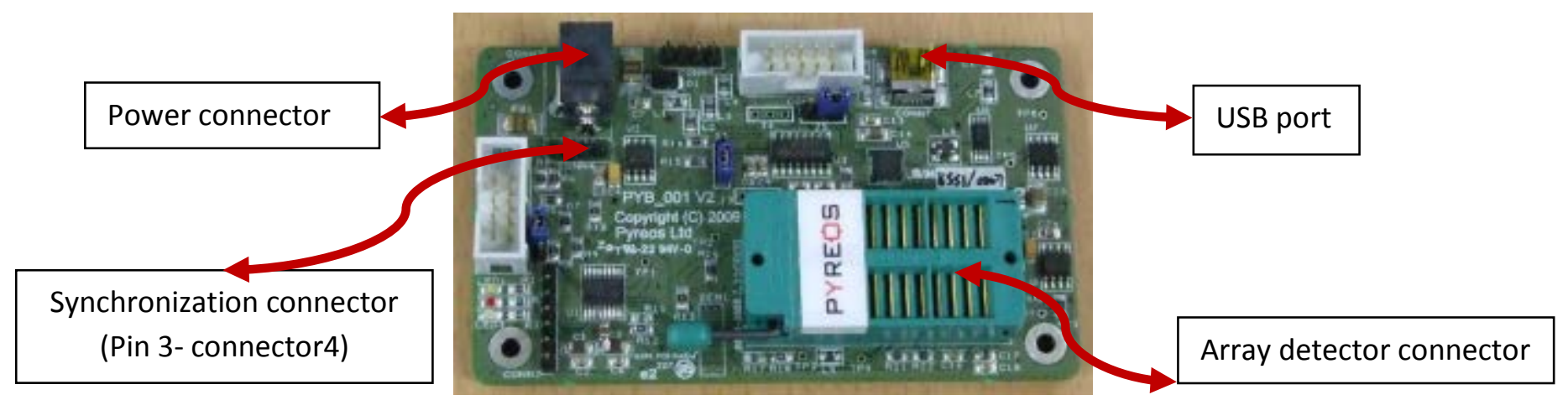

Figure 2: Sensor PCD board.

In an attempt to improve the understanding of the data collected by the detector, some preliminary data were collected only from the room temperature variations. Several modifications were then made to reduce temperature fluctuations due to room lighting. A black box was made to shield as much of the room's light as possible. On the inside of the box, an LED was inserted so most of the change in light recorded by the sensor came from the LED flashes. 
The sensor board is equipped with a set of pins each outputting specific data. Pin 3 of connector 4 shown on Figure 2 out puts the pulse necessary to drive the LED. This pulse is synchronized with the sampling frequency of the detector which can be digitally modified through the software. In the initial setup, an LED was connected and driven by the board. Sets of data were recorded for different sampling frequencies values. The raw data were collected and saved in an EXCEL file. Each column represented a pixel, and each alternating row was the voltage reading of the detector during a period of the data collection time. The data reading happened during two periods; when the LED was on and when it was off

To calculate the actual change in brightness of the LED, an algorithm was created in EXCEL to take the difference between consecutive rows of the EXCEL sheet with each row representing the LED on and LED off recorded values at each pixel. The result of this calculation yielded an array of signal intensities. Later, the average signal for each pixel was taken so a graph of signal intensity vs. pixel could be plotted.

To compare how much noise the room light and other electronics was creating relative to the signal, the average root mean square (RMS) voltage of the signal was calculated from the most lit pixels. From the array of data calculated earlier, the standard deviation of the signal was calculated. The average RMS voltage and the actual signal values were calculated for each frequency and plotted to compare the sensitivity of the detector across a range of frequencies. This RMS signal was then compared to the average signal for each pixel on the same plot. After interpreting the data collected from the initial setup, other experiments were performed. They consisted in covering parts of the detector with regular tape and observing the detectors behavior in different conditions. Figure 3 represents the latest setup used. 


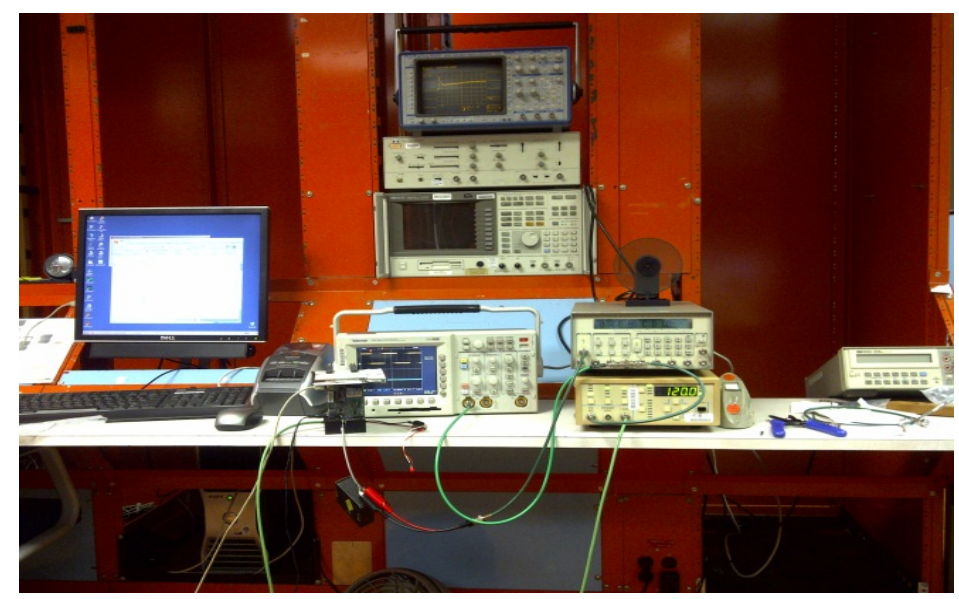

Figure 3: electronics setup to test the detector.

Later in the experiment, a single cell pyroelectric detector (Figure 4) was calibrated for potential use in the alignment of the invisible lasers that will be used in the final design of the spectrometer. The single cell detector was connected to a lock-in amplifier so the current output can be read at a specific frequency. The single cell detector was placed on the optical table after the chopping wheel, a mirror and two lenses used to focus the beam [2] Later, the single cell sensor was replaced by a diode detector that gave information about the power of the beam.

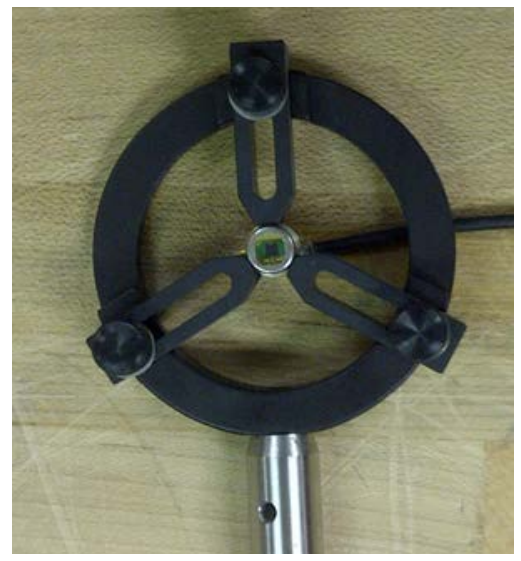

Figure 4: Pyroelectric single cell detector mounted on a holder. 


\section{RESULTS:}

To calibrate the detector, its sensitivity had to be probed by a strong light source, the LED light source was run using the pulse generated from the third pin of the fourth connector of the detector. (Figure 2) It was convenient to use a regular LED light source for this purpose. When using the detector, several anomalies in the data were noticed, indicating some deficiencies in the data collection process. These deficiencies were not easily assigned to the hardware or the software of the detector. To determine the origin of the problems, several tests were performed. These tests yielding data sets that were also challenging to interpret. Figure 5 shows one signal recorded during these tests.

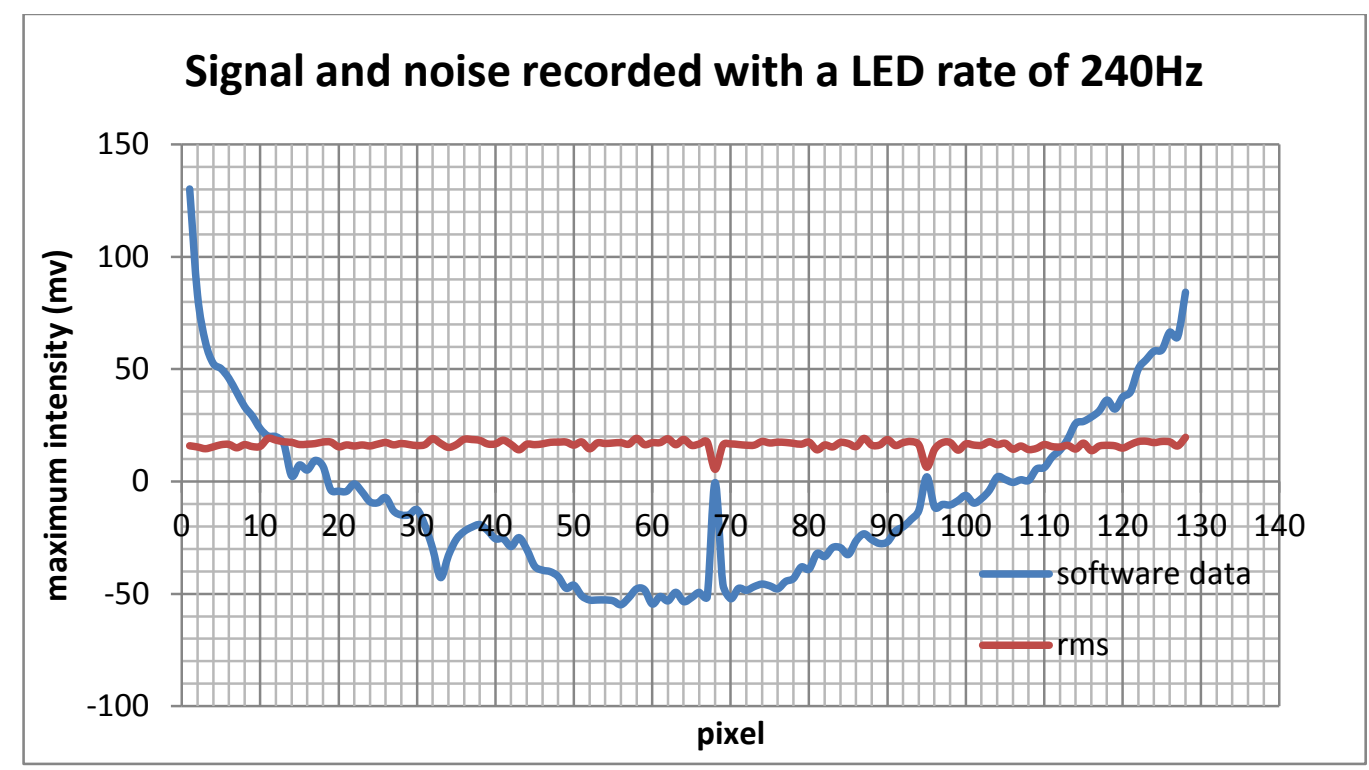

Figure 5: Data collected when running the LED at a frequency of $240 \mathrm{~Hz}$, the LED is placed at the center of the array detector, around pixel 60.

The data shown in Figure 5 represents the output power the sensor recorded when an LED was placed at the center of the array, directly above it. The data yielded an inverted bell-curve. This contrasted with the expectation of a Gaussian distribution. The most intensely-illuminated pixels 
yielded negative intensities, while the least intensely-illuminated pixels yielded positive ones. Also, the whole spectrum represented had an average intensity of $-9.22 \mathrm{mv}$ which is too small to represent the average intensity of the LED. These effects suggest that the detector may be ACCoupled rather than DC-Coupled as described in the company-provided user manual.

These observations were made for each available frequency up to $240 \mathrm{~Hz}$. The data were recorded for frequencies at $10 \mathrm{~Hz}$ intervals.

The manufacturer of the detector was initially contacted in an effort to determine how the detector used raw data. The received information did not fully characterize the observed data, some of which was in direct contradiction to the supplied user manual. The EXCEL algorithm described in the previous section was developed to get a better understanding of the software functioning. Data as the ones produced by the software from the raw data collected from the board were calculated. Then, the noise and the signal were compared for the whole range of frequencies of interest. From that data, the observation that the noise was very small compared to the signal was made. When averaging the signal and noise of the 30 most lit pixels and averaging those for each frequency, we determined that the signal was 43.3 times higher than the noise. Figure 6 shows the plot of the signal compared to the noise. One can notice that the noise is very small relative to the signal. 


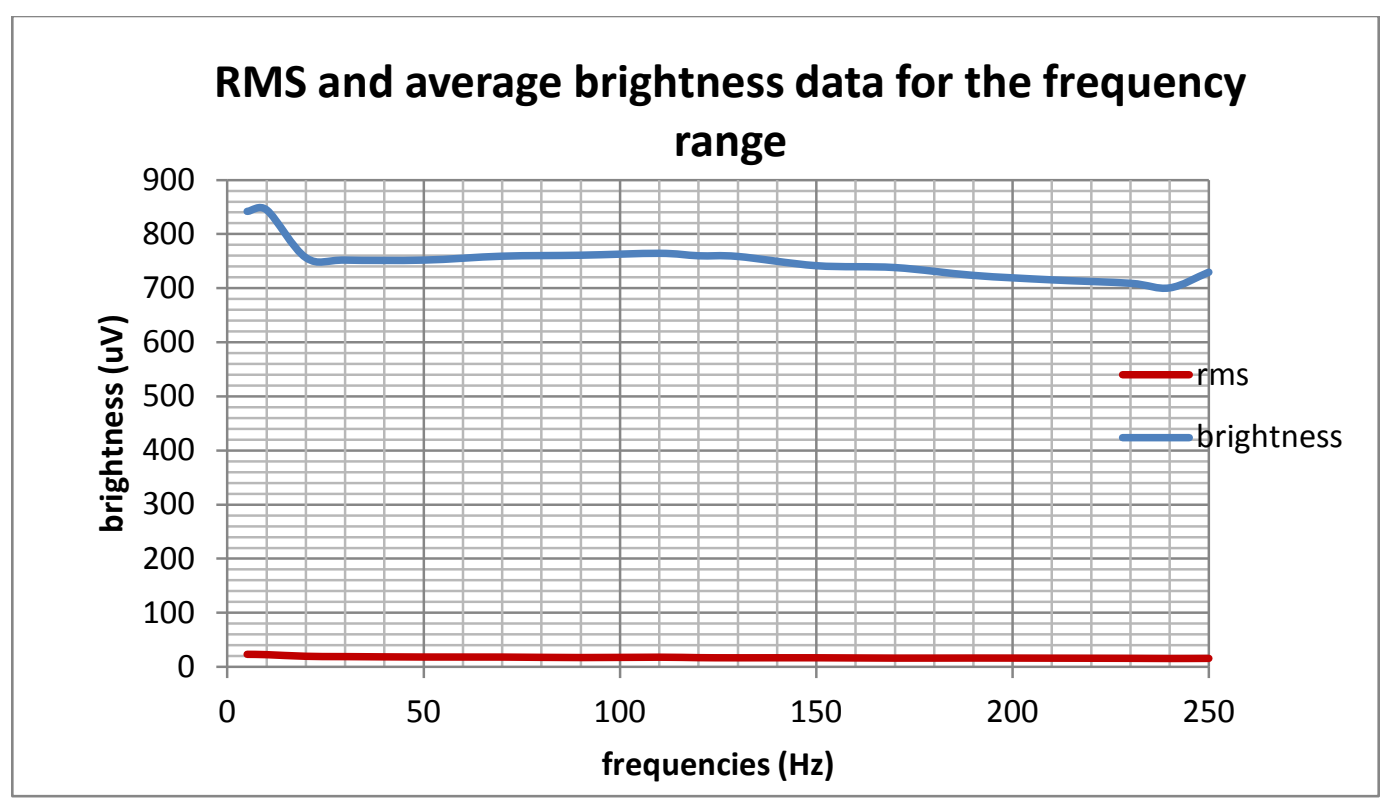

Figure 6: Signal compare to the noise for frequencies from $10 \mathrm{~Hz}$ to $240 \mathrm{~Hz}$.

The software calculations appeared reasonable since the results could be reproduced using a program written in the lab. The only concern now was to know what type of information were the raw data yielding. During this part of the experiment, a dark tape was placed at the center of the array detector. The plot in Figure 7 shows that the detector presented characteristics similar to the initial data collected. These results suggested that the program may have inverted the raw data it received. However, this was inconsistent with the observed behavior of dead pixels on the detector across different tested frequencies. 


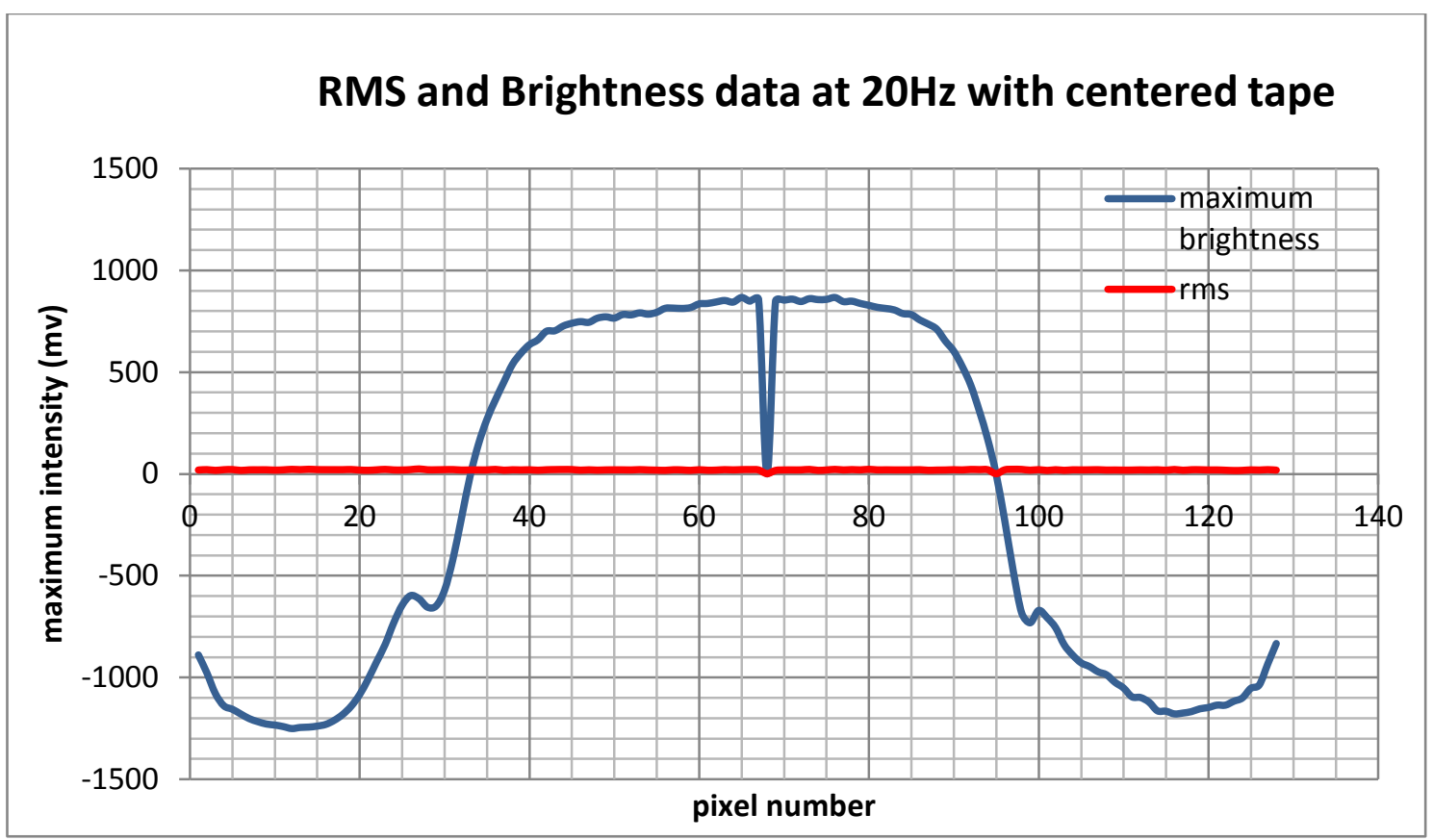

Figure 7: Data collected from the PYREOS sensor with a centered tape, the graph suggests that the software averages the overall signal to zero.

Another issue with the data was observed when the experiment was run at a sampling rate of 220Hz. (Figure 8) instead of a Gaussian curve, the expected inverse bell shaped curve was observed with the minimum brightness at the center of the detector, where the tape was placed. This completely contradicted the according to which the data were just inversed. 


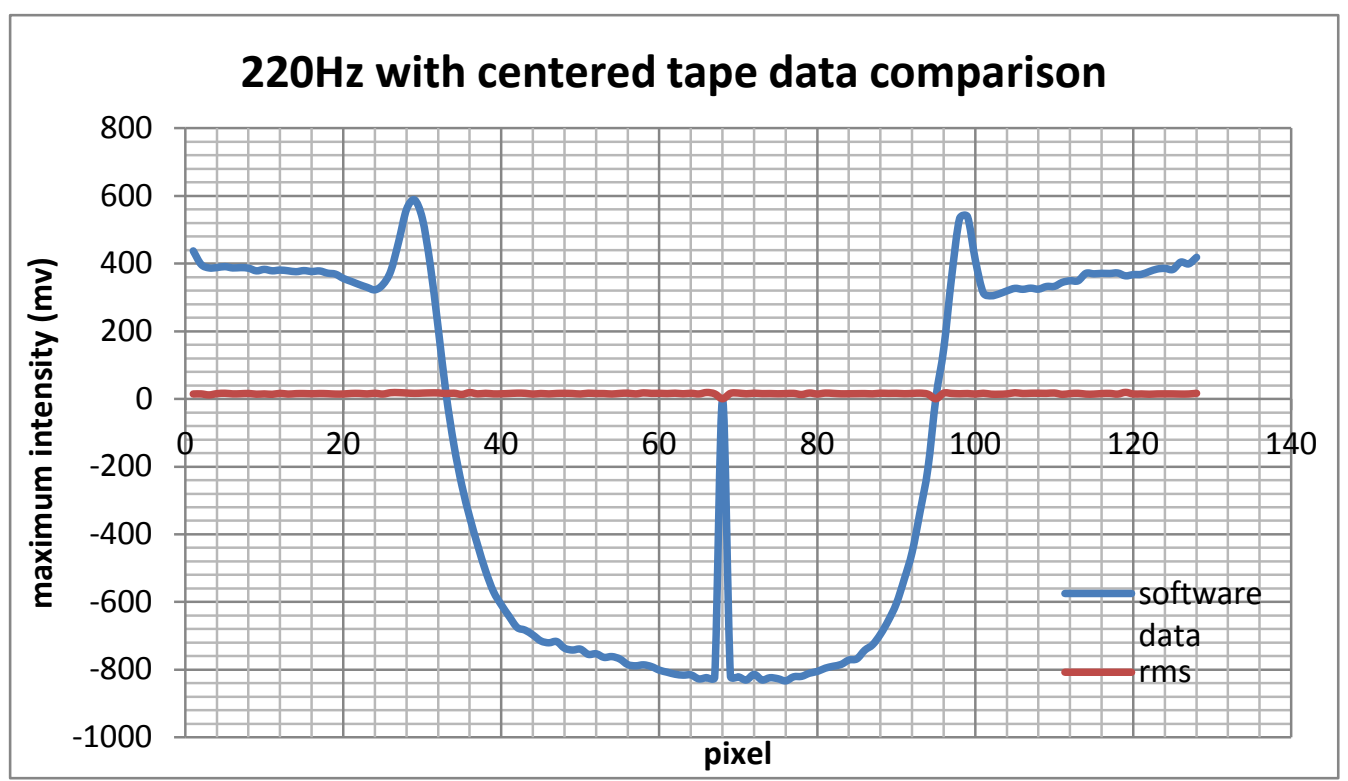

Figure 8: Data collected from the PYREOS sensor at $220 \mathrm{~Hz}$ sampling frequency with a centered tape, the graph suggests that the software averages the overall signal to zero.

In Figure 8, the tape is centered so the observed plot is the shape we expected while the previous data presented the opposite characteristics even though the parameters were the same throughout the experiment. This observation further suggested AC-Coupling in the detector. In this configuration, the slightest change in lightning can result in the change in the whole spectrum. It was suggested that an additional experiment be performed to determine the parameters needed to optimize the detector system. But changing the software parameters and observing the behavior of the detector in real time.

The detector allows for sample rate and data collection interval to both be adjusted. The times that are modifiable are: the 'VVR closed time' (VVR = Voltage VGainStage Reset) and the 'VDR closed time' (VDR = Voltage DGainStage Reset) those two times determine the duration of the detector not recording data: Dead time. Modifying the dead time affected the integration time which is the time during which the detector collects data. 
According to the detector's manufacturer, the integration time is related to the other parameters as follow:

$$
\text { integration time }(u s)=\frac{1}{\text { sample frequency }}(u s)-V D R \text { time }(u s)-3(u s)
$$

Data were collected for a range of sampling frequencies and dead time. Figure 11 shows the table of the data collected, the integration time predicted by the user's manual.

\begin{tabular}{|c|c|c|}
\hline $\begin{array}{c}\text { Sampling } \\
\text { frequencies } \\
\text { (Hz) }\end{array}$ & $\begin{array}{c}\text { VDR time } \\
\text { (us) }\end{array}$ & integration time (us) \\
\hline 240 & 375 & 3788.666667 \\
\hline 240 & 400 & 3763.666667 \\
\hline 240 & 646 & 3517.666667 \\
\hline 230 & 800 & 3544.826087 \\
\hline 220 & 1000 & 3542.454545 \\
\hline 210 & 1200 & 3558.904762 \\
\hline 201 & 1400 & 3572.124378 \\
\hline 197 & 1500 & 3573.142132 \\
\hline 129 & 4000 & 3748.937984 \\
\hline
\end{tabular}

Figure 8: Calculated Integration time for several frequencies to be compared With the delay time measured.

This calculated integration time seemed very high compared to what was observed simultaneously on an oscilloscope (Figure 9). The realization that the time during which the software takes data does not change while the dead time and "the integrating time" decrease was quickly made. 


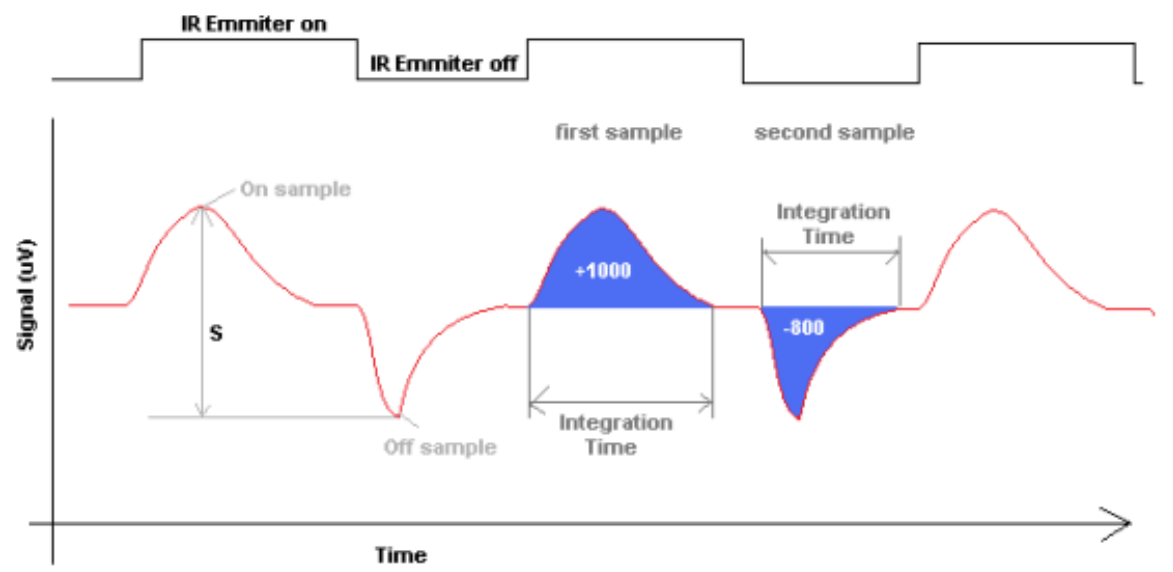

Figure 9: Generated signal and integration time. On this graph from the detector's user's manual, the integration time covers most of the signal period both the on and off phase.

While performing this experiment, it appeared that the integration time was different from the expected time. Using the previously described setup, modifications to the phase difference between the LED pulse and the sampling rate were made. A very small change in this delay time induced using the pulse generator completely nullified the signal when the calculated integration time suggested otherwise. Figure 10 shows how delaying the pulse may cancel the signal, represented by the blue areas under the red curve. This plot shows that the sampling frequency will have to be shifted by a considerable amount for the signal to disappear but continuing the delay will make the signal reappear with a potential change in sign.

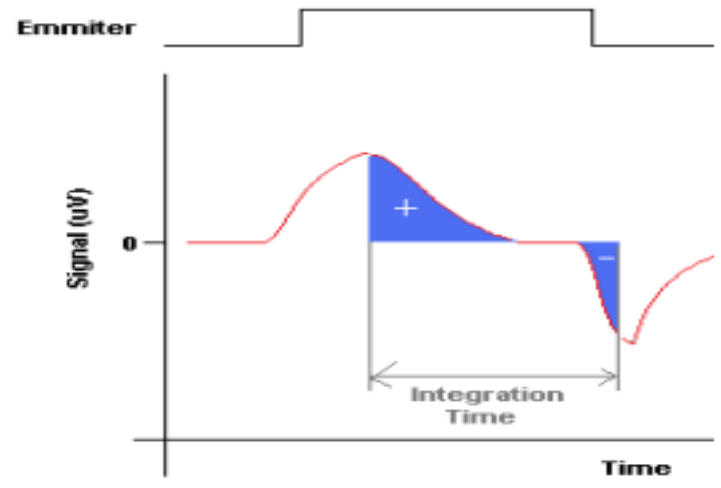

Figure 10: a delay time will result in the cancelation of the signal. 
For the same parameters and dead times as the ones described above, the pulse was delayed in time, and the amount of time it took the detector to lose the signal was recorded. The delay times for several VDR values were recorded in Figure 11 for several frequencies.

\begin{tabular}{|c|c|c|}
\hline $\begin{array}{c}\text { Sampling } \\
\text { frequencies (Hz) }\end{array}$ & $\begin{array}{c}\text { VDR time } \\
\text { (us) }\end{array}$ & delay (us) \\
\hline 240 & 375 & 0 \\
\hline 240 & 400 & 74 \\
\hline 240 & 646 & 258 \\
\hline 230 & 800 & 350 \\
\hline 220 & 1000 & 494 \\
\hline 210 & 1200 & 634 \\
\hline 201 & 1400 & 1270 \\
\hline 197 & 1500 & over \\
\hline 129 & 4000 & over \\
\hline
\end{tabular}

Figure 11: the delay time measured for several frequencies to be compared to the calculated integration time.

Figure 12 shows a plot of both the integration time calculated and the delay time needed for the signal to disappear on the oscilloscope. One can see that the delay time is much too short compared to the integration time to cause the signal to disappear.

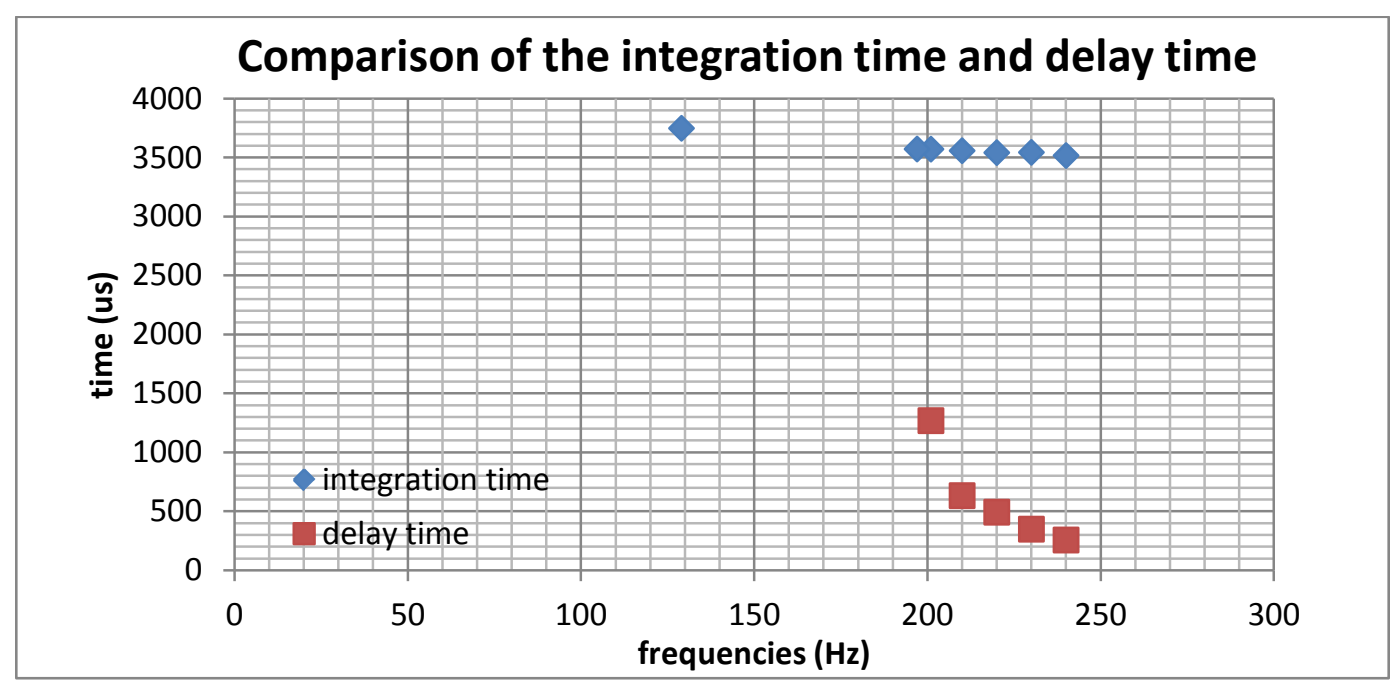

Figure 12: Comparison of the delay time and integration time. One would expect the delay time to be much larger for the signal to disappear. 
Simultaneously, the sensitivity of a single cell, one-pixel pyroelectric detector was measured. A chopper wheel was driven at a frequency of $100 \mathrm{~Hz}$ immediately after the origin of a test laser. The power sensor, placed after the chopping wheel, a mirror, and two lenses to focus the beam, recorded a power output by the laser of $0.531 \mathrm{~mW}$. The same set up was used to determine the current output by the pyroelectric detector; the locking amplifier displayed a current reading of $0.71 \mathrm{nA}$. From this we can infer that the single cell detector has sensitivity of about $1.34 \mathrm{uA} / \mathrm{w}$. at $100 \mathrm{~Hz}$ for the Helium- Neon laser of wave length 633nm.

\section{Discussion and Conclusion:}

The fact that the data is flipped for some frequencies is believed to result from the fact that the program used by the software does not distinguish between subtracting the minimum intensity by the maximum and vice-versa. So one can simply multiply the whole set of data by a -1 and will only have to deal with the AC-Coupling.

The performed test indicated that the detector used provided us with hard to analyze data. We were able to confirm that the issue was not a software deficiency since the program used to get the reading from the raw data was written and gave results identical to the original detector software. This observation, coupled with variation in the data, suggests a hardware deficiency. The detector is thought to be an AC coupled device since the program appears to average the data it receives to 0 when the parameters are changed. Taken together, this evidence suggests a problematic user manual description of the detector integration time. Further experiment with the timing will allow the determination of the integration time for the whole range of frequencies studied. 
Even though a full understanding of the data collected was not achieved, an understanding of its functions and data treatment methods was engaged. With no other candidate for its replacement, understanding the detector's operating scheme is a big step toward the completion of the Infrared spectrometer.

The integration time determination is crucial for the data acquisition phase of the project, and will be a major focus of future work with the detector. The calibration of the sensors depends greatly on the amount of signal collected by the detector, and the right timing must be determined to optimize the reliability of the data. 


\section{Acknowledgement:}

I would like to thank Josef Frisch for being an outstanding mentor; Alan Fisher for his tremendous help and support throughout the project; Mark Petree and Georges Burgess for their assistance in the laboratory work; Stephen Rock for directing the program, all SULI students for the social environment with which they surrounded program. I would also like to thank the department of energy for funding the Science undergraduate Laboratory Internship. Finally, thanks to the SLAC national laboratory and Stanford University for hosting the program.

\section{References:}

[1]Josef Frisch, Tonee Smith, et al, Accelerator physics and engineering update, (Jan 6, 2011) Department updates

[2] C. Behrens, A. F. Design of a single-shet prismspectroeter in the near infrared and mid infrared wavelength range for ultra-short Bunch Length diagnostics. DIPAC'11, Hamburg, Germany, 201

[3] Williams, K.(2011). Optical Design of a Broadband Infrared Spectrometer for Bunch Length Measurement at the Linac Coherent Light Source

[4] Cass, J. (2011). Simulations and Analysis of an Infrared Prism Spectrometer for Ultra-short Bunch Length Diagnostics at the Linac Coherent Light Source.

[5]Alan Fisher, From LCLS to LHC: Light from Electrons, Protons, even Lead ions, (jan 6, 2011) LHC Synchrotron Light monitor, THz studies, Alan Fisher.

[6] Lead Zirconate Titanate http://en.wikipedia.org/wiki/PZT

[7]Tim Chamberlain, (. (2009, december). Thin Film Pyroelectric. Retrieved july 2011, from Pyreos.com:

http://www.pyreos.com/images/docs/PYDK_LAS_Demo_Kit_User_Guide_JWrelease_Jan201 $0 . p d f$

[8]Tim Chamberlain, (n.d.). www.pyreos.com. Retrieved july 1, 2011, from Pyreos Ltd: http://pyreos.com/products/line-sensors.html 